\title{
Parallel proton transfer pathways in aqueous acid-base reactions
}

\author{
M. J. Cox and H. J. Bakker \\ FOM Institute AMOLF, Kruislaan 407, 1098 SJ Amsterdam, The Netherlands
}

(Received 5 October 2007; accepted 6 February 2008; published online 1 May 2008)

\begin{abstract}
We study the mechanism of proton transfer (PT) between the photoacid 8-hydroxy-1,3, 6-pyrenetrisulfonic acid (HPTS) and the base chloroacetate in aqueous solution. We investigate both proton and deuteron transfer reactions in solutions with base concentrations ranging from $0.25 \mathrm{M}$ to $4 M$. Using femtosecond midinfrared spectroscopy, we probe the vibrational responses of HPTS, its conjugate photobase, the hydrated proton/deuteron, and chloroacetate. The measurement of these four resonances allows us to follow the sequence of proton departure from the acid, its uptake by the water solvent, and its arrival at the base. In recent studies it was shown that proton transfer to carboxylate bases proceeds via Grotthuss conduction through a water wire connecting the acid and the base [Mohammed et al., Science 310, 83 (2005);Agnew. Chem. Int. Ed. 46, 1458 (2007);Siwick and Bakker, J. Am. Chem. Soc. 129, 13412 (2007); J. Phys. Chem. B 112, 378 (2008)]. Here we show that, for the weaker base chloroacetate, an alternative channel for proton transfer arises. In this channel the proton is first transferred to the water solvent and only later taken up from the water by the base. We study the base concentration dependence of the two competing channels. (C) 2008 American Institute of Physics. [DOI: 10.1063/1.2889390]
\end{abstract}

\section{INTRODUCTION}

Proton transfer $(\mathrm{PT})$ reactions in aqueous media belong to the most widespread and essential reactions in nature. These reactions are highly complex due to the role that water molecules can play in the reaction mechanism. For example, the transport of just the proton through liquid water is already a quite complicated process. It has been shown with Car-Parrinello molecular dynamics simulations that the proton is transferred through water by a rapid interconversion of Eigen $\left(\mathrm{H}_{9} \mathrm{O}_{4}^{+}\right)$and Zundel $\left(\mathrm{H}_{5} \mathrm{O}_{2}^{+}\right)$solvation structures. ${ }^{1-3}$ This interconversion results in an exchange of positive charge between hydrogen atoms. Hence, only the charge of the proton is transferred in this process, which makes the process very fast and efficient. Recently, the interconversion between Eigen and Zundel proton solvation structures has been observed experimentally with femtosecond midinfrared spectroscopy. 4

A widely used experimental approach to study proton transfer is by monitoring the proton release of so-called photoacids. For these molecules the acidity can be switched by optical excitation, which makes it possible to start the proton transfer reaction at a well-defined point in time. A commonly used photoacid for these types of experiments is pyranine: 8-hydroxy-1,3,6-pyrenetrisulfonic acid trisodiumsalt (HPTS). HPTS has a strong absorption near $400 \mathrm{~nm}$ and can thus be excited using the second harmonic of a Ti:sapphire laser. The excitation leads to an enhancement in the acidity of the molecule by a factor of $10^{6}$. HPTS has been used to study the dynamics of acid dissociation ${ }^{5-11}$ in water using a number of time-resolved spectroscopic techniques. The excited state of HPTS, HPTS*, deprotonates (PT to solvent) with a time constant of $90 / 220$ ps in $\mathrm{H}_{2} \mathrm{O} / \mathrm{D}_{2} \mathrm{O}$.

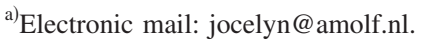

The photoacid HPTS has also been used to study the reaction between an acid and a base dissolved in water. $^{12,14-21}$ If the strength and concentration of the base are sufficient, the release of the proton by HPTS* is strongly accelerated. In this acid-base reaction, the diffusion of the reactants through the water medium can play an essential role, as the reactants have to come sufficiently close to let the proton transfer take place. The above mechanism for intermolecular acid-base reactions has been denoted as the Eigen-Weller framework. ${ }^{22-25}$ An extension of this framework is the so-called Smoluchowski-Collins-Kimball (SCK) model. ${ }^{26,27}$ In this model the acid and the base diffuse to a well-defined mutual distance at which the reaction takes place at a certain reaction rate. The SCK model has been used to describe the proton transfer between HPTS and acetate in $\mathrm{H}_{2} \mathrm{O} / \mathrm{D}_{2} \mathrm{O}$. $^{13}$

Recently, the proton transfer between HPTS and different carboxylate bases dissolved in water has been studied by probing the vibrations of the photoacid and an accepting base with femtosecond midinfrared laser pulses. ${ }^{14-21}$ These studies showed that the SCK model does not provide an adequate description of the data at all delay times. It was also found that there is not a single well-defined reaction distance at which proton transfer takes place. Instead, there exists a distribution of hydrogen-bonded reaction complexes that differ in the number of water molecules separating the acid and the base. ${ }^{14,15,20,21}$ Additionally, at high base concentrations, most of the proton transfers occur in complexes that are already present in the solution before the HPTS molecule is excited. ${ }^{14-21}$ Of course, at low base concentrations, diffusion is important as it is required to generate the hydrogenbonded reaction complexes.

Here we study the mechanism of the proton-transfer reaction between aqueous HPTS and chloroacetate by probing 
the vibrational resonances of the photoacid, the conjugate photobase, the hydrated proton, and the accepting base with femtosecond midinfrared spectroscopy. At high base concentrations, we observe the reaction to be dominated by direct conduction of protons to the base, in agreement with previous work on this system, ${ }^{18}$ and with our findings for the water-acetate system. ${ }^{14,15}$ However, we find that with decreasing base concentration the proton transfer becomes increasingly dominated by another reaction channel. In this channel, the proton is first transferred to water followed by uptake of the proton from the water by a dissolved base ion. We study the competition of this alternative reaction channel with the direct proton conduction channel.

\section{EXPERIMENTAL}

The proton transfer reaction between HPTS and chloroacetate is studied with femtosecond visible pumpmidinfrared probe spectroscopy. The $400 \mathrm{~nm}$ pump pulse is resonant with the absorption band of HPTS that is centered at $365 \mathrm{~nm}$. The excitation of this band switches the $p K_{a}$ of the HPTS molecules from 6 to 0 , thereby initiating an enormous increase in the acidity of the HPTS molecule. The infrared probe pulses are tuned to resonances of the excited HPTS*, the conjugated photobase PTS*-, the hydrated proton/deuteron, and the carbonyl vibration of chloroacetic acid. As a result, all stages of the proton transfer reaction are detected.

The $400 \mathrm{~nm}$ pump pulses and IR probe pulses are generated by nonlinear frequency conversion of light from a regeneratively amplified Ti:sapphire laser (SpectraPhysics Hurricane) that produces $100 \mathrm{fs}, 1 \mathrm{~mJ}$ pulses at $800 \mathrm{~nm}$ at a repetition rate of $1 \mathrm{kHz}$. To generate $400 \mathrm{~nm}$ pump light, we use a type I BBO ( $\beta$-bariumborate) crystal (phase-match angle $\theta=29^{\circ}, 2 \mathrm{~mm}$ ) to double about $20 \%$ of the $800 \mathrm{~nm}$ fundamental. In this process, up to $10 \mu \mathrm{J}$ of $400 \mathrm{~nm}$ light is generated which in most experiments is attenuated to $\sim 1 \mu \mathrm{J}$.

The midinfrared probe pulses are generated through a sequence of nonlinear frequency-conversion processes. The first process of this sequence is a white-light seeded optical parametric amplification stage (SpectraPhysics OPA). The white-light generation and parametric amplification processes are pumped with about $80 \%$ of the output from the laser. The parametric amplification results in two output pulses (signal and idler) that are tunable between 1200 and $1600 \mathrm{~nm}$ (signal), and 1600 and $2400 \mathrm{~nm}$ (idler). The signal and idler are used as input in a difference-frequency mixing process in $\mathrm{AgGaS}_{2}$ (phase-match angle $\theta=45^{\circ}, 2 \mathrm{~mm}$ ). In this process, pulses with wavelengths tunable between 2.7 and $8 \mu \mathrm{m}$ are generated, with a typical pulse duration of $\sim 150 \mathrm{fs}$ and a frequency bandwidth of $200 \mathrm{~cm}^{-1}$.

The samples are solutions of pyranine (8-hydroxy-1,3,6pyrenetrisulfonic acid trisodium salt (HPTS, 98\%) and base sodium-chloroacetate $\left(\mathrm{NaCH}_{2} \mathrm{ClCOO}\right)$ dissolved in $\mathrm{H}_{2} \mathrm{O}$ or $\mathrm{D}_{2} \mathrm{O}$. Both HPTS and $\mathrm{NaCH}_{2} \mathrm{ClCOO}$ were purchased from Aldrich and are used without further purification. In all experiments, the concentration of HPTS was $10 \mathrm{mM}$. The concentration of chloroacetate base was varied $(0,0.25 \mathrm{M}, 0.5 \mathrm{M}$, $1 M, 2 M$, and $4 M$ ). Part of the HPTS (with a maximum of
$20 \%$ at $4 M$ chloroacetate) reacts with chloroacetate to form the conjugate base $\left(\mathrm{PTS}^{-}\right)$and chloroacetic acid, thereby forming self-buffering solutions. A control experiment on a solution containing only PTS $^{-}$showed that the excitation of PTS $^{-}$with the $400 \mathrm{~nm}$ pump pulse does not result in any signal in the midinfrared, except during temporal overlap of the $400 \mathrm{~nm}$ excitation pulse and the midinfrared probing pulses (i.e., for time delays $<200 \mathrm{fs}$ ).

The sample is contained in a rotating cell with $\mathrm{CaF}_{2}$ windows and an optical path length of $50 \mu \mathrm{m}$. The pump pulses are focussed in the sample using a $\mathrm{CaF}_{2}$ lens with a focal length of $20 \mathrm{~cm}$ to a focus with a diameter of about $200 \mu \mathrm{m}$. A 50\% beam splitter splits the IR beam into probe and reference beams. Both probe and reference are focussed into the sample by a parabolic mirror (gold), but only the probe is in spatial overlap with the pump. The transmitted probe and reference pulses are dispersed with an Oriel monochromator and detected by two lines of an Infrared Associates $3 \times 32 \mathrm{MCT}$ (mercury-cadmium-telluride) detector array. The measurement of the reference thus allows for a frequency-resolved correction for shot-to-shot fluctuations in the probe-pulse energy. The pump polarization is rotated to the magic angle using a half-wave plate. As a result, only isotropic absorption changes are detected which implies that the measured signals are not affected by molecular reorientation.

\section{RESULTS AND DISCUSSION}

\section{Experimental results}

An important aspect of our measurements is the ability to observe the vibrational absorption spectrum at variable time delays. The dynamics of the different vibrational resonances reveal the different stages of the proton transfer reaction between HPTS and chloroacetate dissolved in water. At early time delays the signal contains two additional components that show a rapid, pulse-width limited decay. The first of these is a two-photon (visible+infrared) absorption process that requires the pump and the probe pulse to be in temporal overlap. The second contribution is formed by proton transfer reactions in direct contact pairs of HPTS* and chloroacetate. In earlier studies it was shown that the proton transfer in these complexes has a time constant $<200$ fs. ${ }^{16-18}$ For delays greater than $1 \mathrm{ps}$, the two fast signal contributions are no longer present and a much slower nonexponential decay on a time scale of tens to hundreds of picoseconds is observed. In the figures, we plot the signals measured at time delays greater than 1 ps.

Figure 1 shows transient spectra measured for a solution of $10 \mathrm{mM}$ HPTS and $1 M$ of chloroacetate in $\mathrm{D}_{2} \mathrm{O}$ after excitation of HPTS. The spectra in Fig. 1(a) represent the transient response in the frequency region of the aromatic ring vibrations of the excited photoacid HPTS* and its conjugated photobase PTS ${ }^{*-}$. The excitation of HPTS results in a direct change of the absorption of its molecular vibrations. As a result, an instantaneous increase in absorption is observed at 1480 and $1540 \mathrm{~cm}^{-1}$. With increasing delay, these absorption bands decay and a new absorption at $1503 \mathrm{~cm}^{-1}$ grows in 

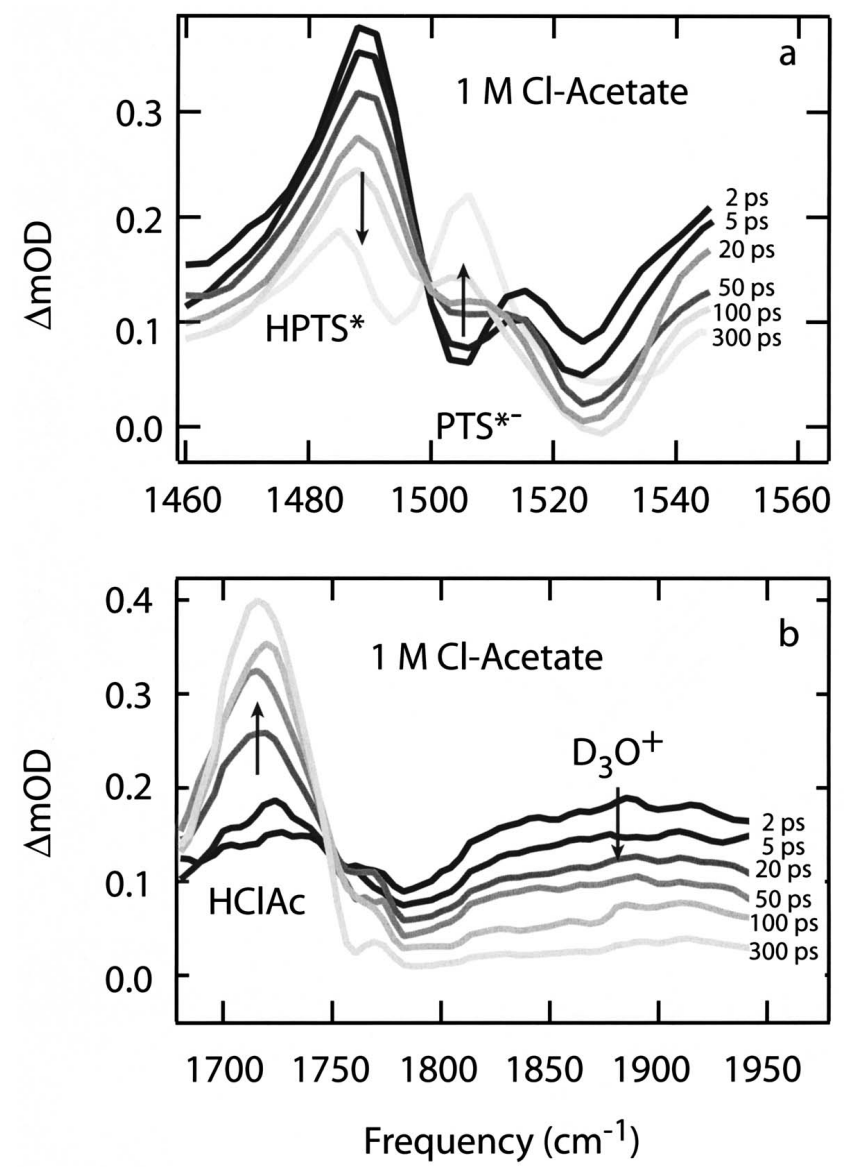

FIG. 1. Transient spectra are shown at six different delays after excitation of a solution of $10 \mathrm{mM}$ HPTS and $1 M$ chloroacetate in $\mathrm{D}_{2} \mathrm{O}$. (a) shows the response of the vibrational bands of HPTS* $\left(1480 \mathrm{~cm}^{-1}\right)$ and the conjugated photo base PTS $^{*^{-}}\left(1503 \mathrm{~cm}^{-1}\right)$. (b) shows the broadband infrared absorption associated with loosely bound and hydrated deuterons $\left(>1800 \mathrm{~cm}^{-1}\right)$, and the absorption band of the carbonyl stretch vibration of chloroacetic acid $\left(1720 \mathrm{~cm}^{-1}\right)$.

that is assigned to the conjugated photobase PTS ${ }^{*-}$. ${ }^{14-21}$ Therefore, the decay and rise of these bands reflect the release of the proton by HPTS*. ${ }^{14-21}$

The transient spectra in the frequency region between 1600 and $2100 \mathrm{~cm}^{-1}$ are shown in Fig. 1(b). This figure shows the rise of the carbonyl stretch absorption of chloroacetic acid centered at $1720 \mathrm{~cm}^{-1}$ [Fig. 1(b)]. The rise of this absorption signals the arrival of the proton/deuteron at the chloroacetate base. In addition to the carbonyl stretch absorption a broadband infrared absorption is observed. This absorption is observed directly after the excitation and decays while the carbonyl stretch absorption rises. The amplitude of the broadband infrared absorption is linear in the pump intensity which implies that this absorption is not the result of multiphoton excitation processes. The broadband infrared absorption can be observed up to the frequency region in which the solution becomes opaque due to the absorption of the stretch vibrations of the solvent, i.e., up to frequencies of $2200 \mathrm{~cm}^{-1}$ in $\mathrm{D}_{2} \mathrm{O}$. Figure 2 shows the broadband infrared response over a wide spectral range for solutions in $\mathrm{H}_{2} \mathrm{O}$. It is seen that in $\mathrm{H}_{2} \mathrm{O}$ the absorption can be observed up to frequencies of $2800 \mathrm{~cm}^{-1}$.

It has been shown before ${ }^{14,15}$ that the broadband infrared
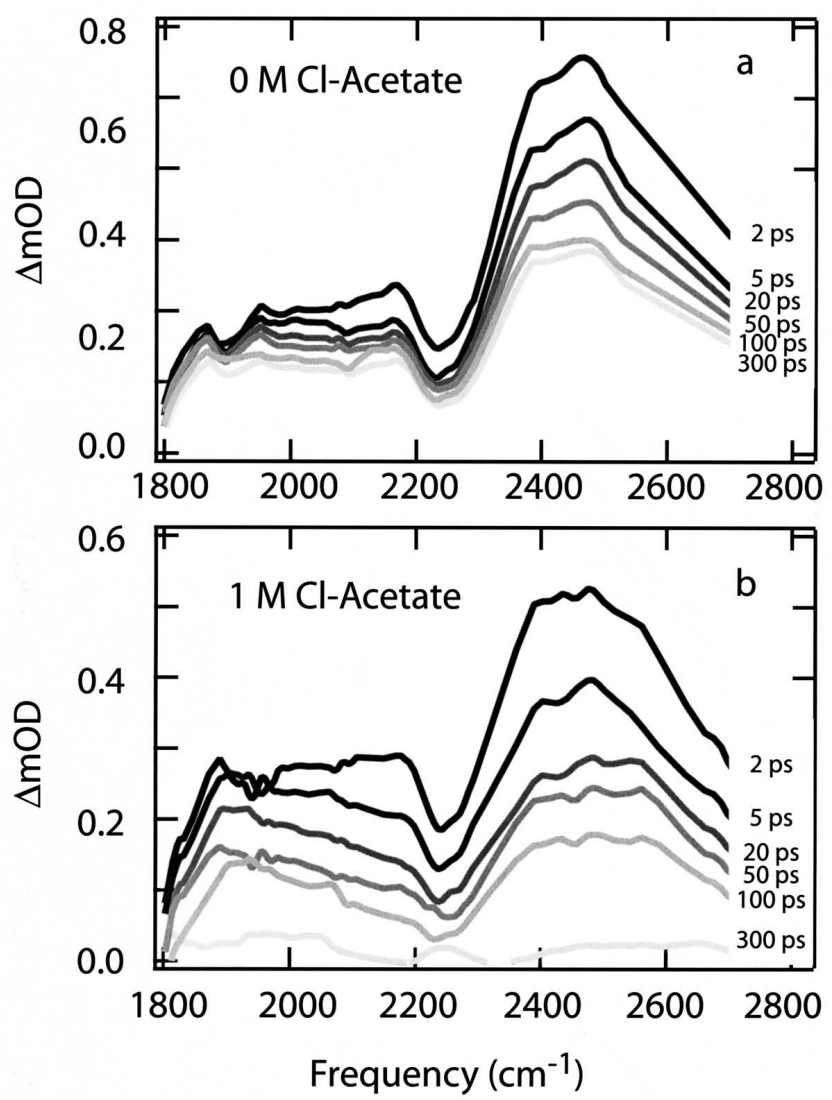

FIG. 2. Transient spectra are shown at six different delays after excitation of HPTS. (a) shows the broadband infrared absorption measured for a solution of $10 \mathrm{mM}$ HPTS in $\mathrm{H}_{2} \mathrm{O}$, with no base present. (b) shows the broadband infrared absorption measured for a solution of $10 \mathrm{mM}$ HPTS and $1 M$ chloroacetate in $\mathrm{H}_{2} \mathrm{O}$.

absorption shown in Figs. 1(b) and 2 represents two different vibrational responses. The first of these responses is associated with the response of the excited state of the HPTS molecule. In this state the proton has not yet left the HPTS molecule. The second of these responses is the vibrational absorption of the fully hydrated proton/deuteron. The broadband nature of the initial absorption of HPTS* indicates that the excitation results in a direct weakening of the $\mathrm{O}-\mathrm{H} / \mathrm{O}-\mathrm{D}$ chemical bond and strengthening of the hydrogen bond to the first solvating water molecule. ${ }^{28-31}$ However, the early time signals of the HPTS* modes near $1500 \mathrm{~cm}^{-1}$ show that in spite of this weakening the $\mathrm{O}-\mathrm{H}$ bond is still intact. This finding agrees with the results of optical studies on the proton transfer dynamics of HPTS and related photoacids. $^{5-11}$

In the case that only HPTS is dissolved in water [no base, Fig. 2(a)], the proton is transferred to the water solvent leading to the formation of hydrated protons. Hence, the spectra shown in Fig. 2(a) at early delay times represent the response of HPTS* with an intact, but weakened $\mathrm{O}-\mathrm{H}$ bond, the spectra at later times represent the response of the fully hydrated proton. In the absence of a base, the signal of the hydrated protons will be very long lived. The signal will eventually decay when the conjugate photobase PTS $^{*-}$ returns to the electronic ground state, thereby becoming a strong base that takes up the proton. This return to the 

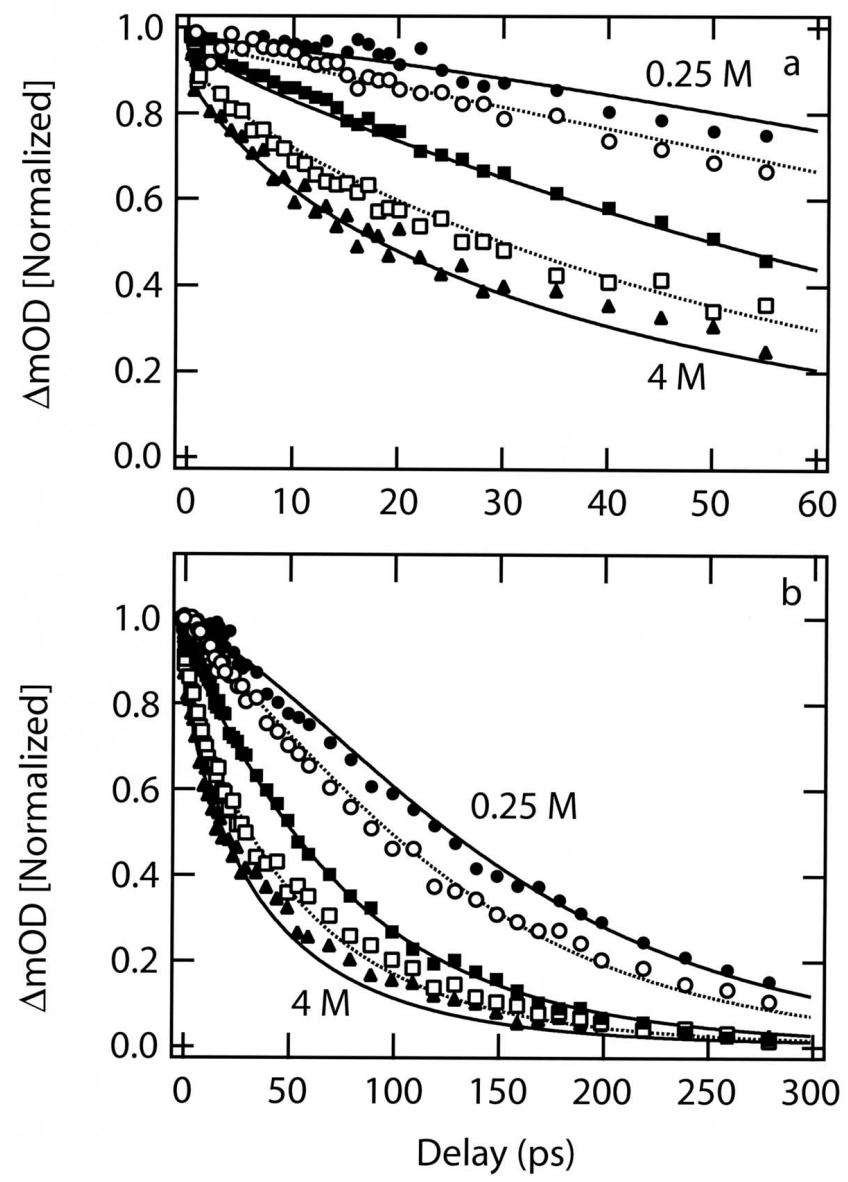

FIG. 3. Normalized responses of the broadband infrared continuum are shown as a function of delay for solutions of $10 \mathrm{mM}$ of HPTS and $0.25 \mathrm{M}$ $0.5 M, 1 M, 2 M$, and $4 M$ of chloroacetate in $\mathrm{H}_{2} \mathrm{O}$. (a) presents the early delay time range from 0 to $60 \mathrm{ps}$, while (b) presents the delay time range from 0 to $300 \mathrm{ps}$. The solid lines are calculated curves using the model described in the text.

ground state takes place on a time scale of $\sim 10 \mathrm{~ns}$. In the presence of the chloroacetate base [Fig. 2(b)], the broadband infrared signal shows a complete decay due to the uptake of the proton by chloroacetate.

Figure 2(a) shows that the transition from the weakened $\mathrm{O}-\mathrm{H}$ bond of HPTS* ${ }^{*}$ to the hydrated proton state is accompanied by a spectral change. At frequencies below $2000 \mathrm{~cm}^{-1}$, the spectral amplitude hardly changes in this transition. At frequencies above $2000 \mathrm{~cm}^{-1}$, the transition results in a partial decay of the amplitude. At a frequency of $\sim 2500 \mathrm{~cm}^{-1}$ the final amplitude is about two times smaller than the initial amplitude. Figure 2 shows that the broadband infrared response contains a broad peak near $2500 \mathrm{~cm}^{-1}$. This broad peak likely represents a proton in an Eigen-type solvation structure. ${ }^{32,33}$ In the case that the proton is loosely bound to HPTS* one of the oxygen atoms in the Eigen-type structure is the oxygen atom of the hydroxyl group of HPTS*.

Figure 3 presents the broadband infrared signal measured at $1900 \mathrm{~cm}^{-1}$ as a function of delay for five different concentrations of chloroaceate dissolved in $\mathrm{H}_{2} \mathrm{O}$. The decay of this signal becomes faster with increasing concentration of chloroacetate. This signal has contributions of both the weakened $\mathrm{O}-\mathrm{H}$ bond of HPTS* and of the fully hydrated
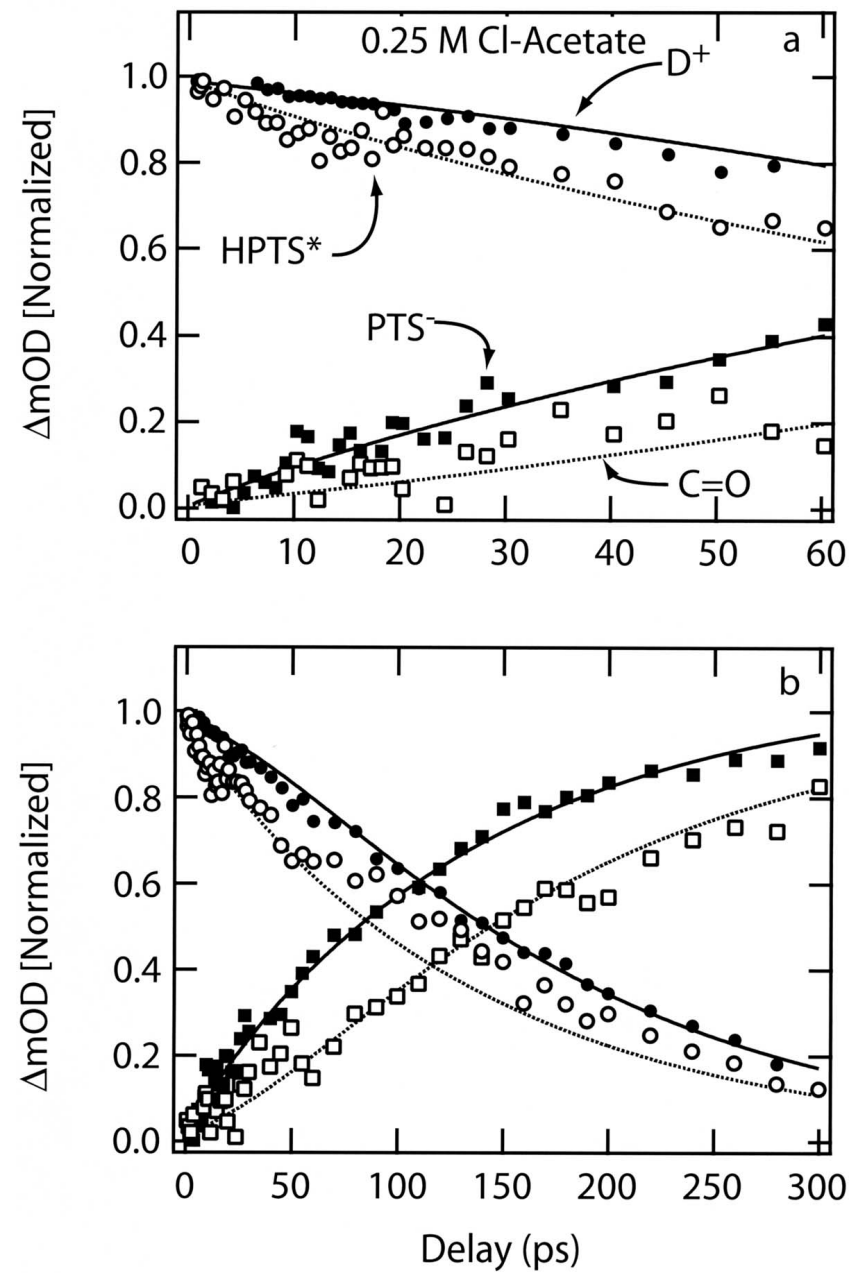

FIG. 4. Normalized responses of HPTS* $\left(1480 \mathrm{~cm}^{-1}\right.$, open circles), the loosely bound and fully hydrated deuteron $\left(1900 \mathrm{~cm}^{-1}\right.$, filled circles $)$, PTS*(1503 $\mathrm{cm}^{-1}$, filled squares), and acetic acid $\left(1720 \mathrm{~cm}^{-1}\right.$, open squares), are shown as a function of delay for a solution of $10 \mathrm{mM}$ HPTS and $0.25 \mathrm{M}$ chloroacetate in $\mathrm{D}_{2} \mathrm{O}$. (a) presents the early delay time range from 0 to $60 \mathrm{ps}$, while (b) presents the delay time range from 0 to $300 \mathrm{ps}$.

proton. At the probe frequency of $1900 \mathrm{~cm}^{-1}$, the transition from HPTS* to fully hydrated protons has very little effect on the magnitude of the signal. Therefore, the decay of the signal represents the decay of the absorption due to the uptake of the proton by the chloroacetate base, irrespective whether the proton is absorbed from HPTS* or rather from the water solvent.

We can obtain detailed information on the reaction mechanism by monitoring the delay dependence of the signals of HPTS, its conjugate photo base PTS ${ }^{*-}$, the proton/ deuteron signal, and the carbonyl stretch of chloroacetic acid at different concentrations. Figure 4 shows delay time scans of the signals corresponding to HPTS*, the loosely bound and fully hydrated deuteron, PTS*- and the carbonyl vibration of chloroacetic acid measured for a concentration of chloroacetate of $0.25 \mathrm{M}$. In this figure we did not include the instantaneous decay of HPTS* and the instantaneous rises of PTS $^{*-}$ and the carbonyl vibration that result from the ultrafast PT reaction that takes place in direct reaction complexes of HPTS and chloroacetate. Figure 4 shows that the rise of the signal associated with $\mathrm{PTS}^{*-}$ and the decay of the 

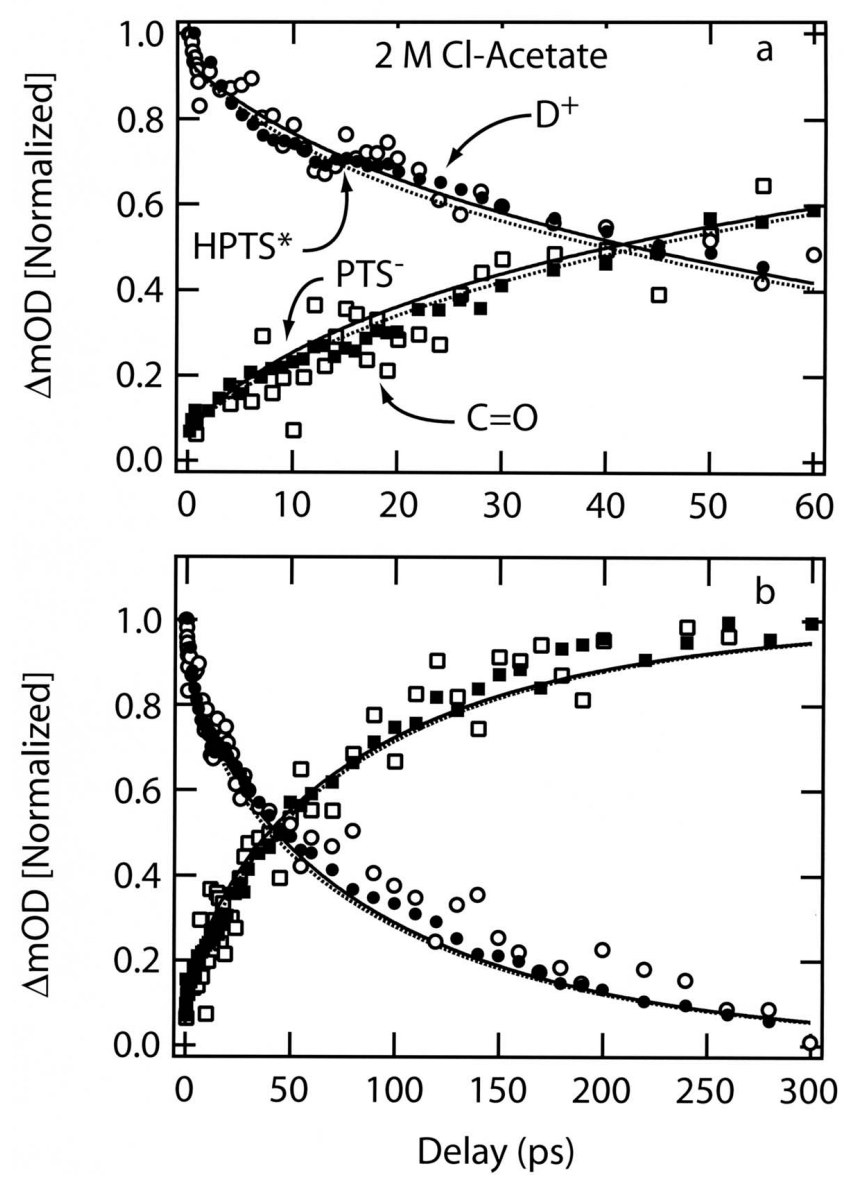

FIG. 5. Normalized responses of HPTS* $\left(1480 \mathrm{~cm}^{-1}\right.$, open circles), the loosely bound and fully hydrated deuteron $\left(1900 \mathrm{~cm}^{-1}\right.$, filled circles), PTS*(1503 $\mathrm{cm}^{-1}$, filled squares), and acetic acid $\left(1720 \mathrm{~cm}^{-1}\right.$, open squares), are shown as a function of delay for a solution of $10 \mathrm{mM}$ HPTS and $2 M$ chloroacetate in $\mathrm{D}_{2} \mathrm{O}$. (a) presents the early delay time range from 0 to $60 \mathrm{ps}$, while (b) presents the delay time range from 0 to $300 \mathrm{ps}$.

signal of HPTS* are much faster than the rise of the carbonyl stretch of chloroacetic acid. This finding implies the deuterons are not directly taken up by the chloroacetate after being released by HPTS $^{*}$, but are first transferred to the water solvent. After some time the deuteron is taken up by the chloroacetate base, leading to the delayed rise of the absorption of the carbonyl vibration of chloroacetic acid.

Figure 5 shows the same four signals as a function of delay measured for a chloroacetate concentration of $2 M$. At this concentration (and at higher concentrations) the rise of the carbonyl vibration of chloroacetic acid shows the same dynamics as the rise of PTS ${ }^{*-}$, which means that there is no delay between the release of the deuteron and the take up of the deuteron by the base, which contrasts strongly with the behavior at lower base concentrations as shown in Fig. 4. It appears that at these higher concentrations deuteron transfer to the water solvent no longer constitutes a significant pathway of the reaction.

From these findings we conclude that the proton transfer involves two reaction channels. In the first channel, the proton is transferred to the water solvent and later absorbed from the solvent by a chloroacetate ion. The transfer to the water solvent is relatively slow: the loosely bound proton/ deuteron has to wait for a characteristic time of 90/220 ps before it is transferred to a fully hydrated state in the $\mathrm{H}_{2} \mathrm{O} / \mathrm{D}_{2} \mathrm{O}$ solvent. The rate at which the proton/deuteron is taken up in the second step from the solvent by a dissolved chloroacetate ion will depend on the concentration of chloroacetate. In the second channel the proton is conducted from HPTS* to chloroacetate in a reaction complex in which HPTS* $^{*}$ and chloroacetate are separated by a small number of intervening water molecules. Such a mechanism has been identified in previous work on proton transfer between HPTS and carboxylate bases. ${ }^{14-21}$ It is to be expected that for small acid-base separations of one or two water molecules the second channel will be much faster than the first channel.

From an experimental point of view the main difference between the two channels is that the first channel involves the temporary uptake of the proton by the water solvent, whereas there is no such uptake in the second channel. As a result, reaction by the first channel leads to a delay between the release of the proton by HPTS and the uptake of the proton by chloroacetate, whereas this delay will be absent for reactions by the second channel. It is also to be expected that at low base concentrations the reaction will be dominated by the first channel, provided that the distribution of acid-base distances is more or less statistical. With increasing base concentrations, the second channel will become increasingly important and at high concentration this channel becomes dominant.

\section{Modeling of the data}

The two parallel reaction channels for proton transfer are included in a model that we fit to the experimental data. This model is an extension of a recently described model for proton transfer via Grotthuss conduction from an acid to a base. $^{14,15}$ The model is extended to describe both channels for proton transfer that we observe in the reaction between aqueous HPTS and chloroacetate. The model also accounts for the mutual diffusion of the reactants.

We consider the solution to consist of configurations of the type

$$
\mathrm{RO}^{-} \cdots \mathrm{H}^{+} \cdots\left(\mathrm{OH}_{2}\right)_{n-1} \cdots \mathrm{B}^{a} \text {, }
$$

where $\mathrm{RO}^{-} \cdots \mathrm{H}^{+}$denotes the acid, $\left(\mathrm{OH}_{2}\right)_{n-1}$ the number of $n-1$ intervening water molecule, and $\mathrm{B}^{a}$ the number of $a$ bases in the $n$th solvation shell. Every water molecule in the connecting chain has two $\mathrm{O}-\mathrm{H}$ groups and can thus donate two hydrogen bonds over which the proton charge can be transferred. Hence, the number of available oxygen positions doubles for every subsequent shell so that shell $n$ contains $2^{n-1}$ oxygen positions.

Each of the oxygen positions in the solvation shells can be occupied by either a water oxygen or an oxygen of the chloroacetate base. The relative fraction of reaction complexes with a base oxygen in a shell with low $n$ will increase with increasing base concentration. We characterize the probability distribution of the different configurations with the function $S[n, a]$, where $n$ is the shell number containing the closest base oxygen atom, and $a$ denotes the number of base oxygen atoms in that shell. We assume the distribution of $S[n, a]$ to be statistical which leads to 


$$
S[n, a]=P_{w}^{\left(2^{n-1}-1\right)}\left(\begin{array}{c}
2^{n-1} \\
a
\end{array}\right) P_{a}^{a} P_{w}^{\left(2^{n-1}-a\right)},
$$

where $P_{w}$ represents the fraction of oxygen atoms in the solution belonging to water molecules and $P_{a}$ represents the fraction of oxygen atoms belonging to chloroacetate ions. The first factor in Eq. (2) represents the probability that the oxygen atoms up to shell $n$ are all water oxygen atoms. The latter factor represents the probability that there are $a$ base oxygen atoms in shell $n$. The values of $P_{w}$ and $P_{a}$ are determined by the concentration of chloroacetate: $P_{a}$ $=2[\mathrm{ClAc}] /\left(\left[\mathrm{H}_{2} \mathrm{O}\right]-2[\mathrm{ClAc}]\right)$ and $P_{w}=1-P_{a}$. The factor of 2 follows from the fact that every chloroacetate contains two oxygen atoms. It can easily be shown that the distribution over the states is normalized: $\Sigma_{n} \Sigma_{a} S[n, a]=1$.

In the first reaction channel the protons are first transferred to the water solvent with rate constant $k_{w}$, leading to a population $H(t)$ of hydrated protons. When the proton is taken up by the base, the population $H(t)$ decreases and a population $C(t)$ of conjugated acid is generated. For low base concentrations, the rate constant of this process will be proportional to the base concentration. We define this rate as $k_{u} P_{a}$, where $k_{u}$ represents the bimolecular rate constant of the proton/deuteron with the base dissolved in water.

In the second reaction channel the proton is conducted within the configurations $S[n, a](t)$. This conduction is only possible when all intervening water molecules possess the correct hydrogen-bond configuration to enable the transfer. The characteristic waiting time for this configuration to arise will increase with the number of intervening water molecules between HPTS and the accepting base. Once an appropriate configuration for conduction is established, the transfer itself has to be a rapid process, as the configurations that allow the transfer are expected to be short lived.

Transfer of the proton via Grotthuss conduction requires that the acid $\mathrm{RO}^{-} \cdots \mathrm{H}^{+}$and the base $\mathrm{B}^{-}$are connected via a wire of $\mathrm{O}-\mathrm{H} \cdots \mathrm{O}$ hydrogen bonds. The conduction involves the concerted transformation of hydrogen bonds into chemical bonds and vice versa in the connecting wire. One of the elements in this chain is the transformation of the chemical bond in RO-H into a hydrogen bond and the formation of a chemical bond to the water molecule in the first solvation shell into a chemical bond: $\mathrm{RO}-\mathrm{H} \cdots \mathrm{O}-\mathrm{H}_{2}$ $\rightarrow \mathrm{RO}^{-} \cdots \mathrm{H}^{+}-\mathrm{O}-\mathrm{H}_{2}$. Another element in the chain is the change of the $\mathrm{O}-\mathrm{H}$ chemical bond in the last water molecule into a hydrogen bond and the transformation of the hydrogen bond to $\mathrm{B}^{-}$into a chemical bond: $\mathrm{O}-\mathrm{H}^{+} \cdots \mathrm{B}^{-} \rightarrow \mathrm{O} \cdots \mathrm{H}-\mathrm{B}$. The rate constant $k[n, a]$ of the conduction will depend on the length of the wire of connecting water molecules. We use the following expression for this rate constant:

$$
k[n, a]=a k_{0} \Delta^{n-2}, \quad n \geqslant 2 .
$$

The parameter $k_{0}$ represents the rate for a reaction complex with only one intervening water molecule between $\mathrm{RO}^{-} \cdots \mathrm{H}^{+}$and the base oxygen. $\Delta$ is a scaling factor by which the rate is multiplied for each additional water molecule. The value of $\Delta$ will be between 0 and 1 . The multiplication with $\Delta$ for each additional intervening water molecule implies that the rate constant $k[n, a]$ decreases exponentially as a function of the number $(n-1)$ of water molecules separating the acid and the base. The reasoning behind this exponential decay is that proton conduction is only possible when all hydrogen bonds in the connecting chain of water molecules allow for the transfer. The rate constant $k[n, a]$ scales with the number $a$ of base oxygens because every chloroacetate oxygen in shell $n$ can take up the proton.

The time dependence of the states $S[n, a]$ is not only determined by proton transfer channels characterized by $k[n, a]$ and $k_{w}$, but also by the mutual diffusion of HPTS and the chloroacetate base. In view of the distance dependence of the rate constant $k[n, a]$, PT will lead to a preferential depletion of the lower shells. The diffusion of base between the shells will thus lead to a net flow of base from the higher shells to the depleted lower shells. As a result, for large initial separations of HPTS and chloroacetate, the chloroacetate likely first hops to a closer shell before it will react and take up the proton. In a recent paper on the HPTS-acetate system, it was described how diffusion can be accounted for. ${ }^{15}$ Including diffusion, we arrive at the following set of coupled differential equations for the states $S[n, a](t), H(t)$, and $C(t)$ :

$$
\begin{aligned}
\sum_{n} \sum_{a=1}^{2^{n-1}} \frac{d S[n, a](t)}{d t}= & -\left(k[n, a]+k_{w}\right) S[n, a](t)-f_{d}[n, a](t) \\
& \times\left[p_{a}[n](t)-p_{a}[n-1](t)\right] \\
& \times k_{i} 2^{n-1} P_{w}^{\left(2^{n-1}-1\right)} \\
& +f_{r}[n, a](t) \sum_{c=1}^{2^{n}} f_{d}[n+1, c](t) \\
& \times\left[p_{a}[n+1](t)-p_{a}[n](t)\right] k_{i} 2^{n} P_{w}^{\left(2^{n}-1\right)} \\
\frac{d H(t)}{d t}=\sum_{n} \sum_{a} k_{w} S[n, a](t)-k_{u} P_{a} H(t) & \\
\frac{d C(t)}{d t}=k_{u} P_{a} H(t), &
\end{aligned}
$$

with

$$
\begin{aligned}
& p_{a}[n](t)=\frac{1}{P_{w}^{\left(2^{n-1}-1\right)} 2^{n-1}} \sum_{a} S[n, a](t) a, \\
& f_{d}[n, a](t)=\frac{a S[n, a](t)}{\sum_{a=1}^{2^{n-1}} a^{2} S[n, a](t)},
\end{aligned}
$$

$$
\begin{aligned}
& f_{r}[n, a](t) \\
& \quad=\frac{\left\{2^{n-1}-(a-1)\right\} S[n, a-1](t)-\left\{2^{n-1}-a\right\} S[n, a](t)}{\sum_{c=1}^{2^{n}} 2^{n-1} S[n+1, c](t)}, \\
& \quad 2 \leqslant a \leqslant 2^{n-1},
\end{aligned}
$$




$$
f_{r}[n, 1](t)=\frac{\sum_{c=1}^{2^{n}} 2^{n-1} S[n+1, c](t)-\left\{2^{n-1}-1\right\} S[n, 1](t)}{\sum_{c=1}^{2^{n}} 2^{n-1} S[n+1, c](t)} .
$$

The coefficients $\quad p_{a}[n-1](t), \quad p_{a}[n](t), \quad p_{a}[n+1](t)$, $f_{d}[n, a](t)$, and $f_{r}[n, a](t)$ depend on partial sums of the states $S[n, a](t)$, thereby coupling the equations for the different $S[n, a](t)$ [Eq. (4)]. For the case $n=1$, the second term in Eq. (4) that represents the net donation of base oxygens to shell $n-1$ vanishes.

A restriction of this model is that the backward reaction from the base and/or water to the photoacid is not included. In the present experiments the inclusion of the back reaction is not required as the photo-acid and the base are relatively strong. For weaker acids/bases the backward proton conduction from base to acid should be included. It is quite straightforward to include the back reaction by redefining $k[n, a]$ as the rate constants for the reaction of states $S[n, a]$ to protontransferred states $A[n, a]$, and by introducing additional distant-dependent reaction rate constants for the reaction of $A[n, a]$ back to $S[n, a]$.

The signals of the vibrational resonances of HPTS*, the conjugated photobase $\mathrm{PTS}^{*-}$, the hydrated proton, and the carbonyl stretch of chloroacetic acid are proportional to the concentrations [HPTS $*](t)$, [PTS $\left.{ }^{*-}\right](t)$, hydrated proton $H(t)$, and $\left[\mathrm{CH}_{2} \mathrm{ClCOOH}\right](t)$, respectively. These concentrations are related to the initially excited concentration $\left[\mathrm{HPTS}^{*}\right](0)$ in the following way:

$$
\begin{aligned}
& {\left[\mathrm{HPTS}^{*}\right](t) /\left[\mathrm{HPTS}^{*}\right](0)=\sum_{n} \sum_{a=1}^{2^{n-1}} S[n, a](t),} \\
& {\left[\mathrm{PTS}^{*-}\right](t) /\left[\mathrm{HPTS}^{*}\right](0)=1-\sum_{n} \sum_{a=1}^{2^{n-1}} S[n, a](t),} \\
& {\left[\mathrm{H}^{+}\left(\mathrm{H}_{2} \mathrm{O}\right)_{n}\right](t) /\left[\mathrm{HPTS}^{*}\right](0)=H(t),} \\
& {\left[\mathrm{CH}_{2} \mathrm{ClCOOH}\right](t) /\left[\mathrm{HPTS}^{*}\right](0)} \\
& \quad=\left\{1-\sum_{n} \sum_{a=1}^{2^{n-1}} S[n, a](t)\right\}-H(t)+C(t) .
\end{aligned}
$$

The time dependencies of the states $S[n, a], H(t)$, and $C(t)$ are calculated by time integrating the coupled equations with a fourth-order Runge-Kutta algorithm. In this calculation, we include 12 solvation shells, i.e., we use a maximum value $n=12$. We take the signal at $1900 \mathrm{~cm}^{-1}$ proportional to the sum of the concentrations [HPTS*] $(t)$ and $\left[\mathrm{H}^{+}\left(\mathrm{H}_{2} \mathrm{O}\right)_{n} / \mathrm{D}^{+}\left(\mathrm{D}_{2} \mathrm{O}\right)_{n}\right](t)$. Here, it should be noted that these two species show the same absorption at $1900 \mathrm{~cm}^{-1}$. We use the values for $k_{w}$ that have been obtained in previous work: $(90 / 220 \mathrm{ps})^{-1}$ in $\mathrm{H}_{2} \mathrm{O} / \mathrm{D}_{2} \mathrm{O}$, and we assume that these values do not change when the base concentration is increased. The only remaining parameters are thus $k_{0}, \Delta$, and $k_{u}$. The values of these parameters are obtained by a simultaneous fit of the model to all the transient signals of HPTS*, the conjugated photo base $\mathrm{PTS}^{*-}$, the proton/deuteron (loosely bound
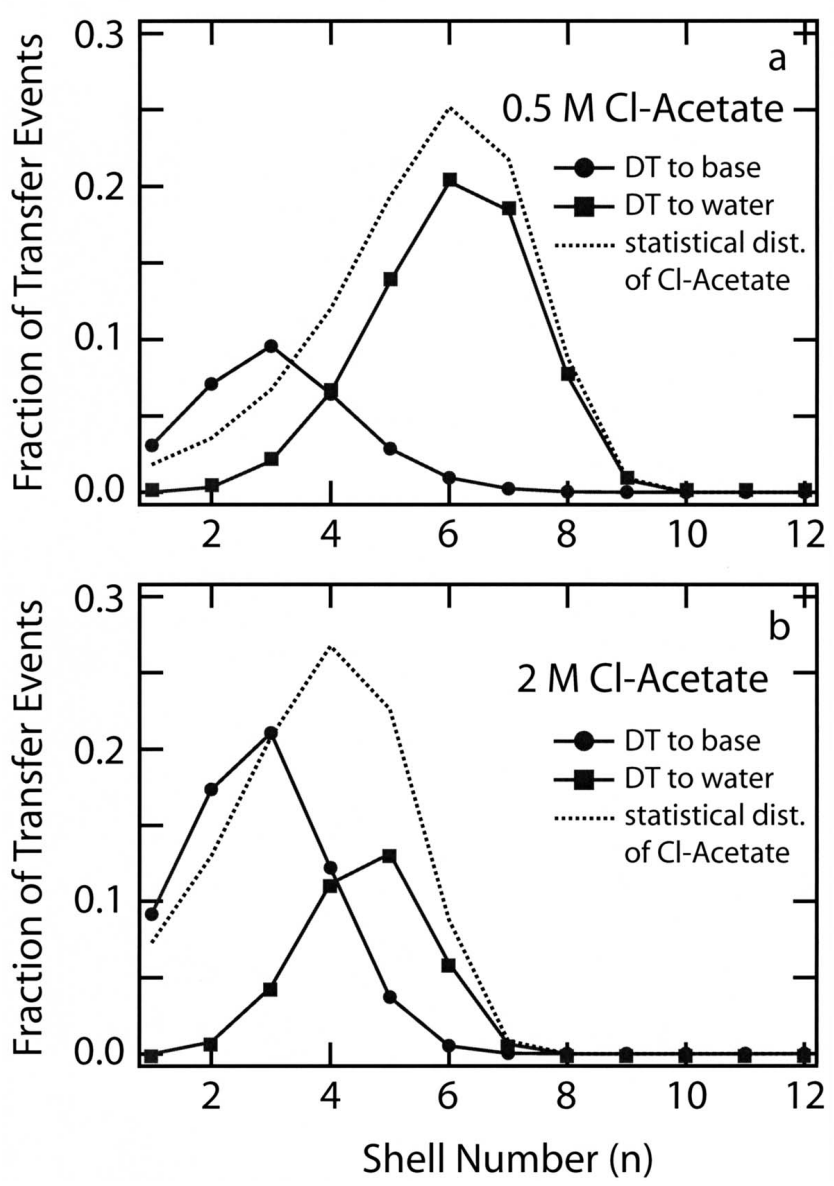

FIG. 6. Fraction of deuteron transfer (DT) events by transfer to the water solvent (solid squares), and direct conduction to the chloroacetate base (solid circles), as a function of solvation shell number $n$. For comparison, the statistical distribution of the chloroacetate over the solvation shells is shown (dashed curve). (a): for a solution of $10 \mathrm{mM}$ HPTS and $0.5 \mathrm{M}$ chloroacetate in $\mathrm{D}_{2} \mathrm{O}$; (b) for a solution of $10 \mathrm{mM}$ HPTS and $2 M$ chloroacetate in $\mathrm{D}_{2} \mathrm{O}$.

+hydrated), and the carbonyl stretch of chloroacetic acid measured at all different chloroacetate concentrations. For the solutions in $\mathrm{H}_{2} \mathrm{O}$, we obtain $k_{0}=0.14 \mathrm{ps}^{-1}, \Delta=0.2$, and $k_{u}=1.5 \mathrm{ps}^{-1}$. For the solutions in $\mathrm{D}_{2} \mathrm{O}$, we obtain $k_{0}$ $=0.10 \mathrm{ps}^{-1}, \Delta=0.2$, and $k_{u}=1.5 \mathrm{ps}^{-1}$. The results of the fits are shown as the solid curves in Figs. 3-5.

Figure 6 shows the fraction of deuteron transfer events for the two reaction channels as a function of the solvation shell number. At a low chloroacetate concentration of $0.5 \mathrm{M}$ [Fig. 6(a)], the reaction is dominated by the transfer to the water solvent, followed by take up of the deuteron from the solvent by the chloroacetate base. The rate of this reaction channel is independent of the distance to the closest chloroacetate base. Therefore, the distribution of deuteron transfers to the solvent closely follows the statistical distribution. Only for the lower solvation shells there are less transfers to the water solvent, because for these shells the competing deuteron conduction reaction channel shows a higher rate. The results shown in Fig. 6(b) show that for the higher base concentration of $2 M$ the majority of the deuteron transfers proceeds through the second reaction channel in which the deuteron is directly transferred to a nearby base. The distribution of deuteron transfers by the second channel clearly differs 
from the statistical distribution: for the lower shells (low $n$ ) the fraction of deuteron transfers exceeds the statistical distribution, for the higher shells (high $n$ ) the fraction is lower than the statistical distribution. This difference in distribution follows from the distance dependence of the reaction rate of the second channel in combination with the presence of diffusion. For reaction complexes in which HPTS and the chloroacetate base are initially separated by many water molecules, the base first diffuses to a lower shell number before the deuteron transfer by conduction takes place. At both $0.5 M$ and $2 M$ chloroacetate, the largest fraction of deuteron transfer via conduction is found for $n=3$, which corresponds to a reaction complex in which HPTS and chloroacetate are separated by two water molecules. However, the distribution is quite broad, and a significant fraction of the deuteron conduction events occurs in reaction complexes in which HPTS and chloroacetate are separated by three $(n=4)$ or even four water molecules $(n=5)$.

\section{Discussion}

The dynamics of the signals at $1900 \mathrm{~cm}^{-1}$ shown in Fig. 3 are observed to be strongly non-exponential at all concentrations. At low base concentrations, the decay of this signal is slow at early delay times and accelerates at later delay times. The initial slow decay is due to the reaction being dominated by the first reaction channel, i.e., most of the proton/deuteron transfers take place to the water solvent. As the hydrated proton has about the same absorption crosssection at $1900 \mathrm{~cm}^{-1}$ as HPTS*, this transfer does not lead to a decay of the signal. However, with increasing concentration of hydrated protons/deuterons the take-up from the solvent by chloroacetate becomes an increasingly likely process. As a result, the decay of the signal at $1900 \mathrm{~cm}^{-1}$ accelerates. From the model calculations it follows that the fraction of fully hydrated protons/deuterons reaches a maximum value of 0.15 at a chloroacetate concentration of $0.25 M$. At $1 M$ of chloroacetate, the maximum fraction of hydrated protons/deuterons is reduced to 0.05 and at $4 M$ it is only 0.008 . The maximum fraction of hydrated protons/ deuterons decreases with increasing base concentration for two reasons. First, the take up rate from the solvent by the base scales with base concentration. Additionally, the competing second reaction channel in which the proton/deuteron is directly conducted to a nearby base will become more important with increasing base concentration. At high base concentrations $(>1 M)$, the reaction is dominated by this second reaction channel. At high base concentrations the decay of the signal at $1900 \mathrm{~cm}^{-1}$ is again nonexponential but now the signal decay decelerates. This nonexponential behavior results from the existence of a distribution of reaction complexes in which the proton/deuteron conduction takes place with different rates. At early delay times the decay is dominated by the fast reaction in closely spaced acid-base complexes, while at longer delays the signal is dominated by the remaining, slower decaying acid-base complexes in which the acid and the base are relatively far apart.

The conduction channel for proton transfer between aqueous HPTS and chloroacetate has been studied before by the groups of Nibbering and Pines. ${ }^{18}$ Here, we study the reaction over a wider range of concentrations to study the competition between the conduction channel and the indirect channel for proton transfer. It is interesting to compare our findings for the conduction channel with the results of Ref. 18. In this latter study a transient narrow absorption band with a width of $\sim 50 \mathrm{~cm}^{-1}$ was observed at $2560 \mathrm{~cm}^{-1}$ for solutions of HPTS and chloroacetate in $\mathrm{H}_{2} \mathrm{O}$ and at $1850 \mathrm{~cm}^{-1}$ for solutions in $\mathrm{D}_{2} \mathrm{O}$. This narrow absorption band was assigned to a relatively long-lived intermediate state $\mathrm{PTS}^{*-} \cdot \cdots \mathrm{H}_{3} \mathrm{O}^{+} \cdots \mathrm{B}$ in which the proton is hydrated by a single water molecule that is embedded between PTS*and chloroacetate. We could not reproduce the narrow resonances observed in Ref. 18. Instead, we observe resonances near 2500 and $1900 \mathrm{~cm}^{-1}$ that are very broad (having a width of $\sim 300 \mathrm{~cm}^{-1}$ ). We assign these broad resonances to HPTS* for which $\mathrm{O}-\mathrm{H}$ bond is weakened and the hydrogen bond to the first solvating water molecule has become stronger. We also find evidence that the proton conduction not only takes place in reaction complexes in which the HPTS and the chloroacetate are separated by 0 or 1 water molecules, but also in complexes in which the reactants are separated by 2,3 , or even 4 water molecules.

The amplitude and dynamics of the broad resonances that we observe also show a different dependence on base concentration from what is observed for the narrow resonances at $2560 / 1850 \mathrm{~cm}^{-1}$ in Ref. 18. The narrow bands were reported to show decay dynamics that are independent of the base concentration, whereas the broad resonances observed here show dynamics that strongly depend on the base concentration (see Fig. 3). The amplitude of the narrow resonances is reported in Ref. 18 to increase linearly with base concentration while the amplitude of the broad resonances that we observe decreases somewhat with increasing base concentration. This latter decrease can be explained from the increased fraction of reactions taking place in direct HPTSchloroacetate complexes.

The values of $k_{0}$ of $0.14 / 0.10 \mathrm{ps}^{-1}$ that we obtain for the proton/deuteron transfer imply that the proton transfer to chloroacetate has an isotope effect of 1.4. This isotope effect is the same as has been observed in the proton transfer from HPTS to acetate. ${ }^{14,15}$ The isotope effect is also the same as that of the transfer of protons/deuterons in neat liquid $\mathrm{H}_{2} \mathrm{O} / \mathrm{D}_{2} \mathrm{O}$, for which it has been demonstrated that the proton transfer involves a Grotthuss type conduction mechanism. $^{1-3}$ The values of $k_{0}$ for the proton/deuteron transfer from HPTS to acetate were found to be $0.83 / 0.56 \mathrm{ps}^{-1}$ for solutions in $\mathrm{H}_{2} \mathrm{O} / \mathrm{D}_{2} \mathrm{O}$ which is about six times larger than the present values found for the proton/ deuteron transfer to chloroacetate. ${ }^{14}$ This difference in proton transfer rate can be explained from the fact that acetate is a significantly stronger base than chloroacetate.

The value of $\Delta=0.2$ is the same as was found before in a study of the proton/deuteron transfer reaction from HPTS to acetate. ${ }^{14}$ This result shows that the value of $\Delta$ is largely determined by the reorganization energy of the solvent, and not by the strength of the base. The value of $\Delta=0.2$ implies that the conduction rate from the acid to the base decreases quite rapidly with increasing number of water molecules in 
the wire connecting HPTS and chloroacetate. The value of $\Delta$ likely represents the probability that the intervening water molecules acquire a near-planar hydrogen-bond structure, as this structure enables the conduction of the proton. ${ }^{1-3}$ In this structure, the hydrogen atoms of the water molecule each donate a hydrogen bond to the oxygen of neighboring water molecule and the oxygen atom accepts only one hydrogen bond. A value of $\Delta=0.2$ implies that the total probability for a water molecule to be three-fold coordinated ( 2 donated, 1 accepted +1 donated, 2 accepted) is approximately $40 \%$. A percentage of $40 \%$ threefold coordinated water molecules implies that each of the four hydrogen bonds of the water molecule has a probability of 0.82 to be intact. Each water molecule can coordinate four hydrogen bonds, which means that the average number of hydrogen bonds equals $4 \times 0.82$ $\approx 3.3$. This number agrees quite well with the average number of hydrogen bonds per water molecule in room temperature liquid water.

In our recent work on the aqueous HPTS-acetate system only the second proton/deuteron conduction reaction channel was observed. ${ }^{14,15}$ The absence of the first indirect reaction channel in that study can be explained from the fact that only relatively high base concentrations $(\geqslant 1 M)$ were studied. In addition, acetate is a stronger base than chloroacetate, making it also more likely that the proton/deuteron is directly conducted to the base instead of being transferred to the water solvent in the first place.

The model we use to describe the parallel proton transfer paths in aqueous intermolecular acid-base reactions can be applied to other bases. The parameter $\Delta$ is largely related to the hydrogen-bond structure of water that is required to conduct the proton. Hence, this parameter should not change for different bases. The parameter $k_{w}$ is related to the direct proton/deuteron transfer to the $\mathrm{H}_{2} \mathrm{O} / \mathrm{D}_{2} \mathrm{O}$ solvent. Therefore, this parameter should also be independent of the chosen base. It is thus to be expected that for another base only the values of $k_{0}$ and $k_{u}$ will change. Conversely, if a different photoacid is used, the parameters $k_{0}$ and $k_{w}$ will change, while $\Delta$ and $k_{u}$ are expected to remain the same.

\section{CONCLUSIONS}

We studied the mechanism of intermolecular aqueous proton/deuteron transfer between the photo-acid 8-hydroxy1,3,6-pyrenetrisulfonic acid (HPTS) and the base chloroacetate with femtosecond vibrational spectroscopy over a wide range of base concentrations. By probing the vibrational responses of the photoacid, the conjugate photobase, the hydrated proton/deuteron, and the accepting base, we record the departure of the proton from the photoacid, its temporary uptake by the water solvent, and its arrival at the base. We find that the proton/deuteron transfer involves two competing reaction channels. In the first channel, the proton is transferred from HPTS* to the water solvent and then taken up from the solvent by a chloroacetate ion. In the second channel, the proton is directly conducted from the excited HPTS* to chloroacetate in a reaction complex in which the acid and the base are connected by a water wire consisting of a few hydrogen-bonded water molecules. The presence of this lat- ter channel agrees with the results obtained in previous work on the HPTS-chloroacetate system ${ }^{18}$ and with the results of our recent study of proton transfer in the aqueous HPTSacetate system. $^{14,15}$

The data can be well described with a conceptually simple model that includes the two reaction channels and diffusion of the reactants. The first indirect channel is described with two parameters: a rate constant $k_{w}$ describing the proton/deuteron transfer rate to the water solvent, and a parameter $k_{u}$ which multiplied with the base molar fraction $P_{a}$ defines the reaction rate of hydrated protons/deuterons with chloroacetate ions in the solution. The reaction rate of the second direct channel is also described with two parameters: a rate constant $k_{0}$ representing the characteristic rate of conduction of the proton over a wire consisting of one water molecule, and a parameter $\Delta$ that defines the decrease of the reaction rate with each additional intervening water molecule. The distribution of reaction complexes is calculated assuming a statistical distribution of chloroacetate over the solvation shells of HPTS.

At concentrations of chloroacetate greater than $1 M$ the reaction is dominated by the second channel, i.e., the direct conduction of protons from HPTS* to a nearby chloroacetate ion. For concentrations of chloroacetate lower than $1 M$ the first channel forms an important alternative reaction channel. The rate of proton/deuteron release from the excited photoacid HPTS* $k_{w}$ is $0.011 / 0.0045 \mathrm{ps}^{-1}$. The value of this rate is taken from previous work on the rate of proton/deuteron transfer from HPTS* to water. The value of $k_{u}$ is $1.5 \mathrm{ps}^{-1}$ which for a solution of $1 M$ chloroacetate leads to a reaction rate of $0.055 \mathrm{ps}^{-1}$. The value of $k_{0}$ is $0.14 / 0.10 \mathrm{ps}^{-1}$ for solutions in $\mathrm{H}_{2} \mathrm{O} / \mathrm{D}_{2} \mathrm{O}$. The isotope effect of the reaction of 1.4 is the same as has been found before for the rate of proton conduction in neat liquid water and for the proton transfer reaction between HPTS* and acetate. ${ }^{14,15}$ The value of $\Delta$ $=0.2$ can be interpreted as the probability that the water molecules acquire the correct hydrogen-bond configuration to conduct the proton. This configuration is likely formed by a threefold coordinated water molecule of which the oxygen atom accepts only one hydrogen bond.

\section{ACKNOWLEDGMENTS}

The research presented in this paper is part of the research program of the Stichting Fundamenteel Onderzoek der Materie (Foundation for Fundamental Research on Matter) and was made possible by financial support from the Nederlandse Organisatie voor Wetenschappelijk Onderzoek (Netherlands Organization for the Advancement of Research).

${ }^{1}$ D. Marx, M. E. Tuckerman, J. Hutter, and M. Parrinello, Nature (London) 397, 60 (1999).

${ }^{2}$ P. L. Geissler, C. Dellago, D. Chandler, J. Hutter, and M. Parrinello, Science 291, 2121 (2001).

${ }^{3}$ H. Lapid, N. Agmon, M. K. Petersen, and G. A. Voth, J. Chem. Phys. 122, 014506 (2005).

${ }^{4}$ S. Woutersen and H. J. Bakker, Phys. Rev. Lett. 96, 138305 (2006).

${ }^{5}$ E. Pines, D. Huppert, and N. Agmon, J. Chem. Phys. 88, 5620 (1988).

${ }^{6}$ N. Agmon, E. Pines, and D. Huppert, J. Chem. Phys. 88, 5631-5638 (1988).

${ }^{7}$ K. M. Solntsev, D. Huppert, N. Agmon, and L. M. Tolbert, J. Phys. 
Chem. A 104, 4658 (2000).

${ }^{8}$ T.-H. Tran-Thi, T. Gustavsson, C. Prayer, S. Pommeret, and J. T. Hynes, Chem. Phys. Lett. 329, 421 (2000).

${ }^{9}$ J. T. Hynes, T.-H. Tran-Thi, and G. Granucci, J. Photochem. Photobiol., A 154, 3-11 (2002).

${ }^{10}$ P. Leiderman, L. Genosar, and D. Huppert, J. Phys. Chem. A 109, 5965 (2005).

${ }^{11}$ D. B. Spry and M. D. Fayer, J. Chem. Phys. 127, 204501 (2007).

${ }^{12}$ E. Pines, B.-Z. Magnes, M. J. Land, and G. R. Fleming, Chem. Phys. Lett. 281, 413 (1997)

${ }^{13}$ L. T. Genosar, B. Cohen, and D. Huppert, J. Phys. Chem. A 104, 6689 (2000).

${ }^{14}$ B. J. Siwick and H. J. Bakker, J. Am. Chem. Soc. 129, 13412 (2007).

${ }^{15}$ B. J. Siwick, M. J. Cox and H. J. Bakker, J. Phys. Chem. B 112, 378 (2008)

${ }^{16}$ M. Rini, B.-Z. Magnes, E. Pines, and E. T. J. Nibbering, Science 301, 349 (2003).

${ }^{17}$ M. Rini, D. Pines, B.-Z. Magnes, E. Pines, and E. T. J. Nibbering, J. Chem. Phys. 121, 9593 (2004).

${ }^{18}$ O. F. Mohammed, D. Pines, J. Dreyer, E. Pines, and E. T. J. Nibbering, Science 310, 83 (2005).

${ }^{19}$ O. F. Mohammed, D. Pines, J. Dreyer, E. Pines, and E. T. J. Nibbering,
ChemPhysChem 6, 625 (2005).

${ }^{20}$ O. F. Mohammed, D. Pines, E. T. J. Nibbering, and E. Pines, Angew. Chem., Int. Ed. 46, 1458 (2007)

${ }^{21}$ O. F. Mohammed, D. Pines, E. Pines, and E. T. J. Nibbering, Chem. Phys. 341, 240 (2007).

${ }^{22}$ M. Eigen, W. Kruse, G. Maass, and L. de Maeyer, Progress in Reaction Kinetics, (Pergamon, New York, 1964), Vol. 2, p. 285.

${ }^{23}$ M. Eigen, Angew. Chem., Int. Ed. Engl. 3, 1 (1964).

${ }^{24}$ A. Weller, Z. Elektrochem. 58, 849 (1954).

${ }^{25}$ A. Weller, Z. Phys. Chem. 17, 224 (1958).

${ }^{26}$ F. C. Collins and G. E. Kimball, J. Colloid Sci. 4, 425 (1949).

${ }^{27}$ S. A. Rice, Diffusion-Limited Reactions (Elsevier, Amsterdam, 1985).

${ }^{28}$ A. Witkowski, J. Chem. Phys. 47, 3645 (1967).

${ }^{29}$ Y. Marechal and A. Witkowski, J. Chem. Phys. 48, 3637 (1967).

${ }^{30}$ S. Bratos, J. Chem. Phys. 63, 3499 (1975).

${ }^{31}$ B. Boulil, O. Henri-Rousseau, and P. Blaise, Chem. Phys. 126, 263 (1988).

${ }^{32}$ J. Kim, U. W. Schmitt, J. A. Gruetzmacher, G. A. Voth, and N. E. Scherer, J. Chem. Phys. 116, 737 (2002).

${ }^{33}$ J. M. Headrick, E. G. Diken, R. S. Walters, N. I. Hammer, R. A. Christie, J. Cui, E. M. Myshakin, M. A. Duncan, M. A. Johnson, and K. D. Jordan, Science 308, 1765 (2005). 